\title{
Low doses of ionizing radiation induce immune-stimulatory responses in isolated human primary monocytes
}

\author{
HOUSSEIN EL-SAGHIRE ${ }^{1,2}$, ARLETTE MICHAUX ${ }^{1}$, HUBERT THIERENS $^{2}$ and SARAH BAATOUT ${ }^{1,3}$ \\ ${ }^{1}$ Radiobiology Unit, Molecular and Cellular Biology, Belgian Nuclear Research Centre (SCK-CEN), \\ $2400 \mathrm{Mol} ;{ }^{2}$ Department of Basic Medical Sciences, Faculty of Medicine and Health Sciences; \\ ${ }^{3}$ Department of Molecular Biotechnology, Ghent University, 9000 Ghent, Belgium
}

Received July 29, 2013; Accepted September 2, 2013

DOI: $10.3892 /$ ijmm.2013.1514

\begin{abstract}
The health effects arising from exposure to low doses of ionizing radiation are of particular concern, mainly due to the increased application of diagnostic and therapeutic X-ray modalities. The mechanisms behind the cell and tissue responses to low doses remain to be elucidated. Accumulating evidence suggests that low doses of ionizing radiation induce activation of the immune response; however, the processes involved have yet to be adequately investigated. Monocytes are key players in the induction of an immune response. Within the context of this study, we investigated the activation of toll-like receptors (TLRs), mitogen-activated protein kinases (MAPKs) and NF- $\kappa \mathrm{B}$ signaling in isolated human primary monocytes in response to low doses (0.05 and $0.1 \mathrm{~Gy})$ and a high dose (1 Gy) of ionizing radiation. Using quantitative RT-PCR and ELISA techniques, our results showed a positive regulation of TLR signaling in response to low doses but a less significant response at high doses. This activation was demonstrated via the activation of TLR signaling molecules (HMGB1, TLR4, TLR9, MyD88 and IRAK1). Furthermore, and in contrast to the high dose, the low doses showed increased phosphorylation levels of the protein IкB $\alpha$, and therefore positive signaling of the NF- $\kappa \mathrm{B}$ pathway. This result denotes pro-survival and pro-inflammatory responses. Additionally, MAPKs were activated in response to $0.05 \mathrm{~Gy}$, while 0.1 and 1 Gy showed a downregulatory trend that may be related to activation of the PF4 gene. On the other hand, there was highly significant involvement of activated p53 and damaged genes in response to high but not low doses. In conclusion, this study addressed the need to re-evaluate health risks arising from exposure to low doses of ionizing
\end{abstract}

Correspondence to: Dr Houssein El-Saghire, Radiobiology Unit Molecular and Cellular Biology, Belgian Nuclear Research Centre (SCK-CEN), Boeretang 200, Mol 2400, Belgium

E-mail: hesaghir@sckcen.be

Key words: low dose, ionizing radiation, human primary monocytes, inflammatory response, NF- $\kappa \mathrm{B}$, MAPK, TLR, high dose, damage radiation, particularly in view of the accumulating evidence reporting inflammatory and oncogenic consequences from these exposures.

\section{Introduction}

There is increasing concern regarding the health risks arising from exposure to low doses of ionizing radiation, particularly within the medical diagnostic and therapeutic fields $(1,2)$. One of the challenging factors in the study of the health effects of exposures to low doses of ionizing radiation is the lack of epidemiological data, primarily due to confounding factors related to inter-individual variability, life style, and genetic background (3). Furthermore, in contrast to the well-studied mechanisms and the thoroughly investigated health effects of high doses, where cancer is a well-validated endpoint, the mechanisms and the biological responses, induced by low doses of ionizing radiation, are largely not agreed upon in the published studies (4).

Previously, we demonstrated that low doses of ionizing radiation induce immune responses in whole blood samples, with the gene set enrichment analysis showing the involvement of both innate and adaptive immunity (5). Monocytes are key players in the immune response. These cells originate from the bone marrow and are then released into the bloodstream following maturation, while in tissues, they differentiate into macrophages and myeloid dendritic cells (DCs) (6). Monocytes are important in antigen presentation, release of immune-regulatory cytokines, T-cell stimulation and differentiation, thus orchestrating the immune system in response to 'attacking signals' (7). Toll-like receptors (TLRs) are crucial in initiating an immune response in monocytes. TLRs are involved in connecting the innate and adaptive immune responses together and activating monocytes to secrete pro-inflammatory cytokines $(8,9)$. In humans, there are 10 characterized TLRs (TLR 1-10). They interact with evolutionary conserved pathogen-associated molecular patterns (PAMPs), such as lipopolysaccharides (LPS), but also with endogenous stress signals, known as danger-associated molecular patterns (DAMPs), including fibrinogen, heat shock proteins, high mobility group B1 (HMGB1) protein, as well as extracellular matrix components (10). Four adapter proteins play a key role in the signaling of TLR pathways: MyD88, 
Tirap, Trif and Tram. These pathways activate the signaling of the transcription factors NF- $\mathrm{kB}$, activator protein 1 (Ap-1) and interferon factors (IRF) 3 or 7. MyD88 pathway is the major signaling adapter, and is involved in the promotion of NF- $\mathrm{KB}$ translocation into the nucleus and activation of mitogen-activated protein kinases, leading to growth factors and cytokine secretion $(8,11)$.

In this study, we investigated the activation of different TLRs in response to low and high doses of ionizing radiation by measuring the expression of TLR2, TLR9 as well as TLR4, a key monocyte TLR. The three receptors were chosen as they share a common DAMP (HMGB1), and due to the mounting evidence of their involvement in response to radiation stresses (12-14). Furthermore, the investigation included downstream signaling pathways; in particular, MAPKs, a group of serine/threonine protein kinases involved in a wide variety of cell processes, such as cell differentiation, proliferation, apoptosis, as well as in the secretion of inflammatory cytokines. There are three major MAPK pathways: the extracellular-signal-regulated-kinase (ERK), the Jun $\mathrm{N}$-terminal kinase (JNK) and the $\mathrm{p} 38$ pathways (15). We also evaluated the phosphorylation levels of $\mathrm{I} \kappa \mathrm{B} \alpha$, a protein that sequesters NF- $\mathrm{B}$ in the cytoplasm and prevents its translocation to the nucleus (16). The results showed an immune-stimulatory response at low doses of ionizing radiation through the involvement of TLR4 axis of signaling (HMGB1-TLR4-MyD88-IRAK1). This observation was confirmed by the activation of MAPK and NF- $\kappa \mathrm{B}$ signaling. By contrast, high doses predominantly exhibit a damaging response, through the induction of classical ionizing radiation damage and stress genes (CDKN1A, POLH,DDB2 and AEN) and activation of the p53 protein. Furthermore, the immune-suppressive response was demonstrated, mainly via the suppression of NF- $\kappa \mathrm{B}$ and MAPK signaling.

\section{Materials and methods}

Monocyte isolation, culture and irradiation. After signing an informed consent form, $48 \mathrm{ml}$ of peripheral blood was obtained from 8 healthy donors in EDTA vacutainer tubes. The whole blood samples were divided into three different $50 \mathrm{ml}$ Falcon tubes ( $16 \mathrm{ml} /$ tube), and $160 \mu 1$ of $100 \mathrm{mM}$ EDTA and $800 \mu \mathrm{l}$ of Rosettesep ${ }^{\mathrm{TM}}$ Human Monocyte Enrichment Cocktail (StemCell $^{\mathrm{TM}}$ Technologies, Vancouver, BC, Canada) were added to each tube, according to the manufacturer's instructions. Blood was diluted at 1:1 with phosphate-buffered saline (PBS) (Gibco-BRL, Ghent, Belgium) supplemented with 2\% heat-inactivated fetal bovine serum (FBS) (Gibco-BRL) and $1 \% 100$ mM EDTA (Invitrogen Life Technologies, Carlsbad, CA, USA). The diluted whole blood samples $(32 \mathrm{ml})$ were transferred into SepMate ${ }^{\mathrm{TM}}-50$ tubes (StemCell ${ }^{\mathrm{TM}}$ Technologies) and layered over $15 \mathrm{ml}$ of density gradient medium, FicollPaque $^{\mathrm{TM}}$ PLUS (GE Healthcare, Uppsala, Sweden), according to the manufacturer's instructions. SepMate ${ }^{\mathrm{TM}}-50$ (StemCell ${ }^{\mathrm{TM}}$ Technologies) tubes were centrifuged at 1,200 $\mathrm{x}$ g for $10 \mathrm{~min}$ at room temperature. The top layer, containing the monocytes, was transferred into a new tube and washed three times with the washing buffer. All the washing steps were performed at $350 \mathrm{x}$ g for $8 \mathrm{~min}$ with brakes off. The cells were counted using a Moxi Z Mini Automated Cell Counter (Orflo Technologies,
Hailey, ID, USA). Cells $\left(1.2 \times 10^{6}\right)$ were then cultured in each of the 6-well plates (Grenier Bio-One, Wemmel, Belgium) at a concentration of $5 \times 10^{5}$ cells $/ \mathrm{ml}$ of RMPI- 1640 medium (Gibco-BRL) supplemented with 20\% heat-inactivated FBS (Gibco-BRL) and 1\% penicillin-streptomycin $(100 \mathrm{U} / \mathrm{ml}$ penicillin, $0.1 \mathrm{mg} / \mathrm{ml}$ streptomycin) (Invitrogen Life Sciences, Ghent, Belgium). The culture plates were exposed at room temperature to sham irradiation (control) or to three different X-ray doses $(0.05,0.1$ and $1 \mathrm{~Gy})$, at a rate of $30 \mathrm{mGy} / \mathrm{min}$ $(250 \mathrm{kV}, 1.6 \mathrm{~mA}, 1 \mathrm{~mm} \mathrm{Cu})$. Subsequently, the cultured plates were incubated for $6 \mathrm{~h}$ at $37^{\circ} \mathrm{C}$ in a humidified incubator with $5 \% \mathrm{CO}_{2}$.

RNA isolation, $c D N A$ synthesis and quantitative RT-PCR $(q R T-P C R)$. Adherent cells were scraped using Mini Cell Scrapers (Biotium, Hayward, CA, USA) and washed three times with PBS (Gibco-BRL). All the washing steps were performed at $350 \mathrm{x}$ g for $8 \mathrm{~min}$, then suspended in $350 \mu 1$ of lysis buffer (RLT buffer $+10 \mu \mathrm{l} / \mathrm{ml}$ of $\beta$-mercaptoethanol) provided in the RNeasy ${ }^{\circledR}$ Mini Kit (Qiagen, Germantown, MD, USA). Lysates were stored at $-80^{\circ} \mathrm{C}$ until processed. RNA was isolated using the RNeasy ${ }^{\circledR}$ Mini Kit (Qiagen) following the manufacturer's instructions. RNA quantity was measured using a NanoDrop 2000 Spectrophotometer (Thermo Scientific, Aalst, Belgium). The quality was assessed with Agilent 2100 Bioanalyzer (Agilent Technologies, Santa Clara, CA, USA), and average RIN was $8.9 \pm 0.5$. For qRT-PCR experiments, we selected six different genes belonging to TLR signaling (HMGB1, TLR4, TLR9, TLR2, IRAK1 and MyD88) and four genes belonging to damage response (CDKN1A, $P O L H, D D B 2$ and $A E N$ ), as well as the PF4 gene, shown to be involved in MAPK signaling. RPLPO was used as a housekeeping gene. Briefly, cDNA was prepared from $200 \mathrm{ng}$ of total RNA using SuperScript ${ }^{\circledR}$ Vilo $^{\mathrm{TM}}$ Master Mix (Invitrogen Life Technologies) following the manufacturer's instructions. RT-PCR was performed using TaqMan ${ }^{\circledR}$ Gene Expression Assays (Applied Biosystems, Grand Island, NY, USA). Each assay comprised two gene-specific primers and TaqMan assay: FAM (6-carboxyfluorescein) labeled with MGB (minor groove binder) probe. Each TaqMan assay was run in duplicate for each diluted cDNA sample using TaqMan ${ }^{\circledR}$ Fast Advanced Master Mix (Applied Biosystems). The reactions were run on 7500 Fast Real-Time PCR system (Applied Biosystems) following the manufacturer's recommended PCR program: $95^{\circ} \mathrm{C}$ for $20 \mathrm{sec}$, followed by 40 cycles of $95^{\circ} \mathrm{C}$ for $3 \mathrm{sec}$ and $60^{\circ} \mathrm{C}$ for $30 \mathrm{sec}$. Relative expression values were calculated by the Pfaffl method (17).

Protein extraction, quantification and ELISA assay. Adherent cells were scraped using Mini Cell Scrapers (Biotium) and washed three times with PBS (Gibco-BRL). All the washing steps were performed at $350 \mathrm{x}$ g for $8 \mathrm{~min}$, then lysed with Lysis Mix provided in Abcam PhosphoTracer kits (Abcam ${ }^{\circledR}$, Cambridge, UK) following the manufacturer's instructions. Proteins were quantified using Quick Start ${ }^{\mathrm{TM}}$ Bradford Protein Assay (Bio-Rad, Hercules, CA, USA) following the manufacturer's instructions. Proteins were stored at $-80^{\circ} \mathrm{C}$ until assays were performed. Quantitative measurements of the proteins of interest were performed using a PhosphoTracer ELISA kit (ab119661) for the measurement of phosphorylated IкB $\alpha$ 

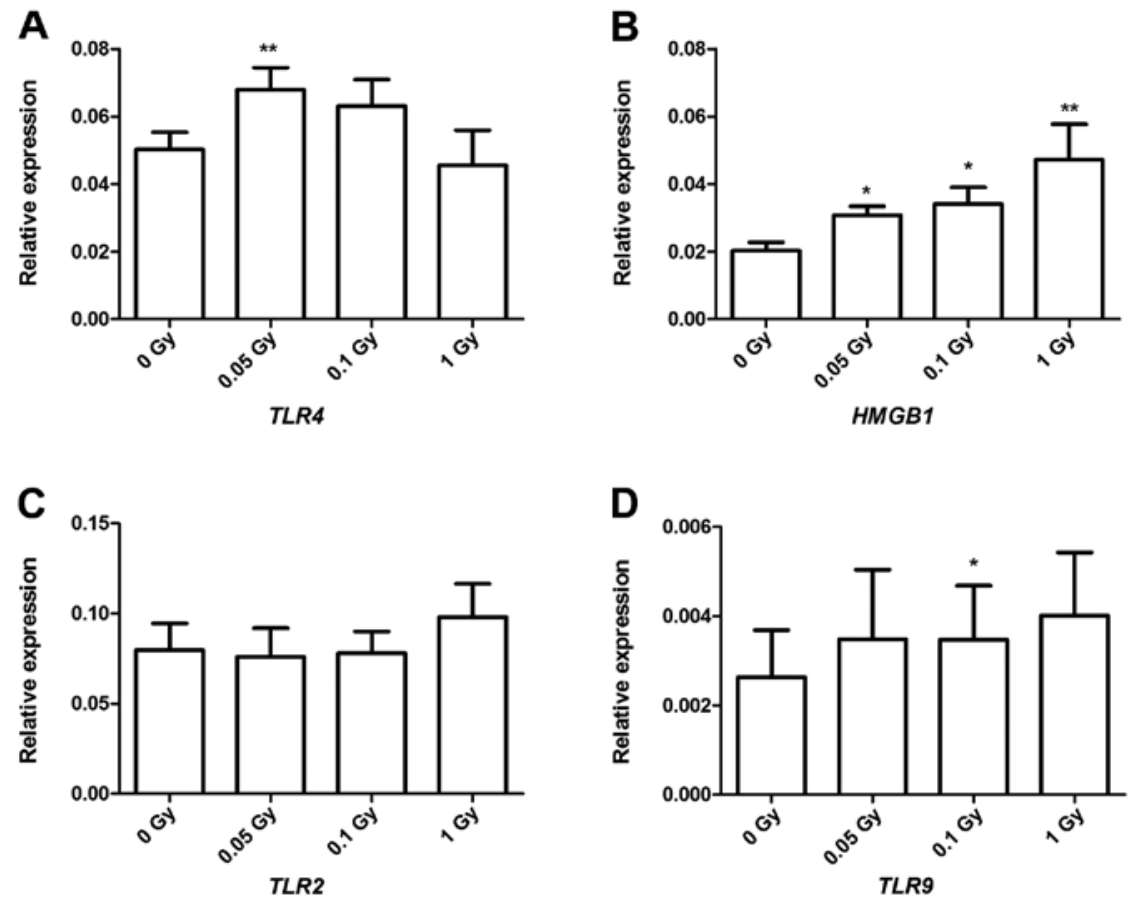

Figure 1. Toll-like receptors and HMGB1 are activated in response to ionizing radiation. Comparative qRT-PCR results of (A) TLR4, (C) TLR2 and (D) TLR 9 and their interaction molecule (B) HMGB1 in response to low and high doses of ionizing radiation. The chart shows the mean values and the standard error of the mean. Statistical significance was calculated by applying the Friedman test followed by Dunn's multiple comparison test. $\mathrm{P}<0.05$ was considered statistically significant. ${ }^{*} \mathrm{P}<0.05 ;{ }^{* *} \mathrm{P}<0.005$.

(Ser32/36) $\left(\right.$ Abcam $\left.^{\circledR}\right)$, PhosphoTracer ELISA kit (ab119674) for the measurement of phosphorylated ERK (Thr202/Tyr204), p38 MAPK (Thr180/Tyr182) and JNK 1/2/3 (Thr202/Tyr185) $\left(\mathrm{Abcam}^{\circledR}\right)$ and PhosphoTracer ELISA kit (ab119666) for the measurement of phosphorylated p53 (Ser15). The immunosandwich ELISA assay was performed according to the manufacturer's instructions. Fluorescence signals were measured using the microplate reader Fluoroskan Ascent CF (Thermo Labsystems, Franklin, MA, USA) with an excitation filter of $544 \mathrm{~nm}$ and an emission filter of $590 \mathrm{~nm}$. To obtain protein fold changes, the fluorescence signals from irradiated samples were normalized to their matching sham-irradiated controls.

Statistical analysis software and graphical representation. For statistical analysis, normality was checked using the D'Agostino and Pearson omnibus normality test. Statistical significance was estimated using the Friedman test or repeated measures ANOVA and Dunn's or Tukey tests for multiple comparison. $\mathrm{P}<0.05$ was considered to indicate statistical significance. Statistical analysis and graphical representation were performed using the GraphPad Prism 5 program.

\section{Results}

Cultured monocytes. Monocytes were isolated and purified from human whole blood samples using density gradient centrifugation and human monocyte enrichment antibody cocktail. The average number of purified cells was $375 \pm 83 \times 10^{3}$ cells $/ \mathrm{ml}$ whole blood. Results of the trypan blue staining showed that at least $95 \%$ of the cells cultured for $6 \mathrm{~h}$ were viable.
Low doses of ionizing radiation induced the TLR signaling axis. Monocytes were activated via the induction of TLR signaling. Our results showed a significant upregulation of TLR4 at 0.05 Gy (Fig. 1A). Furthermore, there was a significant upregulation of TLR9 at 0.1 Gy (Fig. 1B). TLR2 did not show any significant changes in response to any of the doses (Fig. 1D). TLR2, 4 and 9 interact with the endogenous molecule HMGB1, which is secreted by activated monocytes or released due to increased damage. All the doses showed increased levels of HMGB1 (Fig. 1C).

Adapter molecules are important in initiating the signal transduction of the activated TLRs. One of the key adapter molecules is MyD88. TLR 2, 4 and 9 signaling is known to be mediated via MyD88. Although our results demonstrated an upregulatory trend for all the doses, only the samples irradiated with 0.05 Gy achieved statistical significance (Fig. 2A). IRAK1 interacts with MyD88 for further promotion of TLR signaling, leading to the activation of NF- $\kappa \mathrm{B}$ and MAPKs. Our results showed an upregulation in the levels of IRAK1 at 0.05 and $0.1 \mathrm{~Gy}$, but not at $1 \mathrm{~Gy}$ (Fig. 2B).

Low doses of ionizing radiation induced positive $N F-\kappa B$ signaling and MAPK activation. IRAK1 is known to interact with $\mathrm{I} \kappa \mathrm{B} \alpha$, which is a protein that sequesters the NF- $\kappa \mathrm{B}$ transcription factor in the cytoplasm and prevents its translocation to the nucleus. Following activation of NF- $\kappa \mathrm{B}$ signaling, I $\mathrm{I} \mathrm{B} \alpha$ is phosphorylated and degraded. Phosphorylation at Ser32/36 is essential for the release of NF- $\kappa \mathrm{B}$ and it is considered a good marker for $\mathrm{NF}-\kappa \mathrm{B}$ activation. Our results showed upregulation in the phosphorylation of $\mathrm{I} \kappa \mathrm{B} \alpha$ at 0.05 and $0.1 \mathrm{~Gy}$ and downregulation in the phosphorylation levels at $1 \mathrm{~Gy}$ (Fig. 3A). In addition to that, MyD88-IRAK1 signaling molecules promote 
A

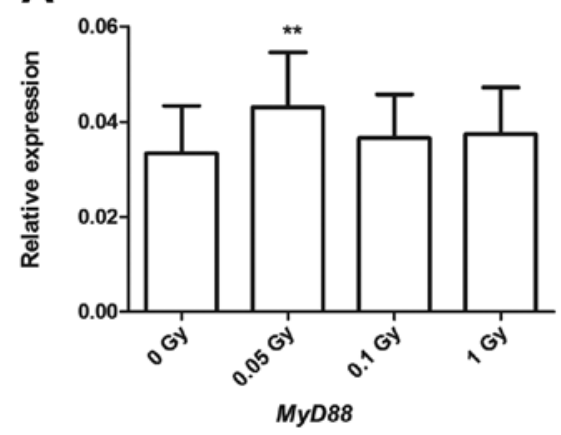

B

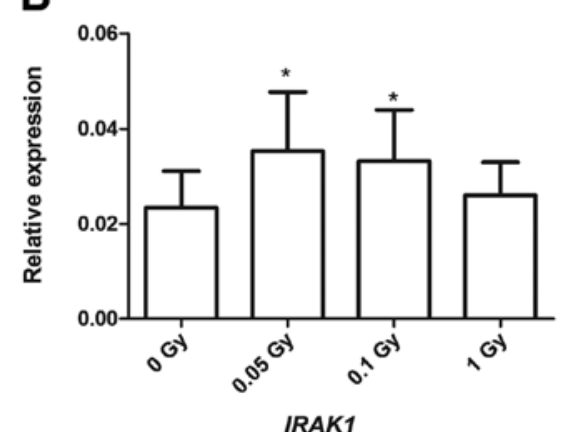

Figure 2. Molecules downstream of TLRs were activated in response to low doses of ionizing radiation. Comparative qRT-PCR results of the adapter molecule $M y D 88$ (A) and IRAK1 (B). The chart shows the mean values and the standard error of the mean. Statistical significance was calculated by applying the Friedman test followed by Dunn's multiple comparison test. $\mathrm{P}<0.05$ was considered statistically significant. ${ }^{*} \mathrm{P}<0.05{ }^{*}{ }^{* *} \mathrm{P}<0.005$.

A

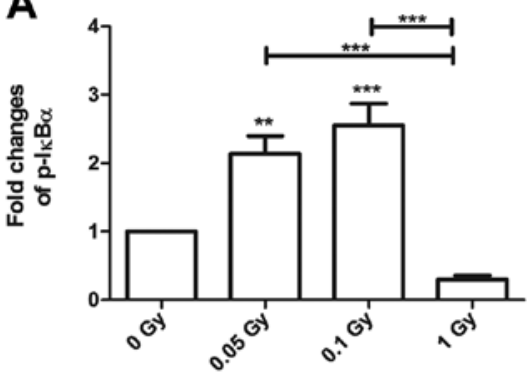

C

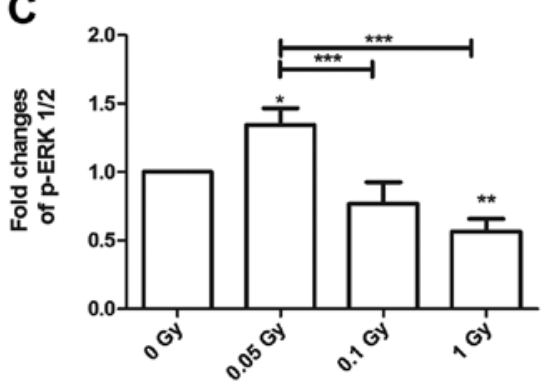

B

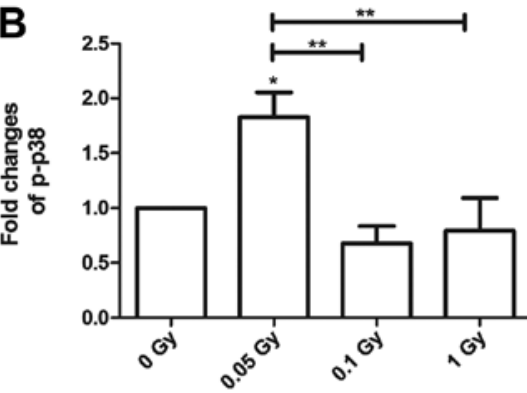

D

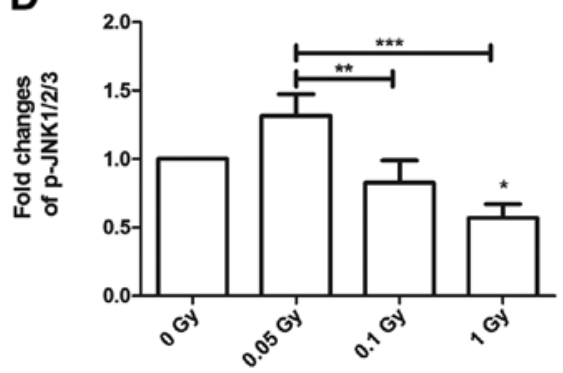

Figure 3. Low doses of ionizing radiation induced pro-survival and pro-inflammatory responses. Fold changes of the phosphorylated forms of the proteins (A) I $\mathrm{B} \alpha$, , (B) p38, (C) ERK 1/2 and (D) JNK 1/2/3. The chart shows the mean values and the standard error of the mean. Statistical significance was calculated by applying one-way repeated measures ANOVA followed by the Tukey's test for multiple comparisons. P<0.05 was considered statistically significant. ${ }^{*} \mathrm{P}<0.05 ;{ }^{* *} \mathrm{P}<0.005 ;{ }^{* * *} \mathrm{P}<0.0001$.

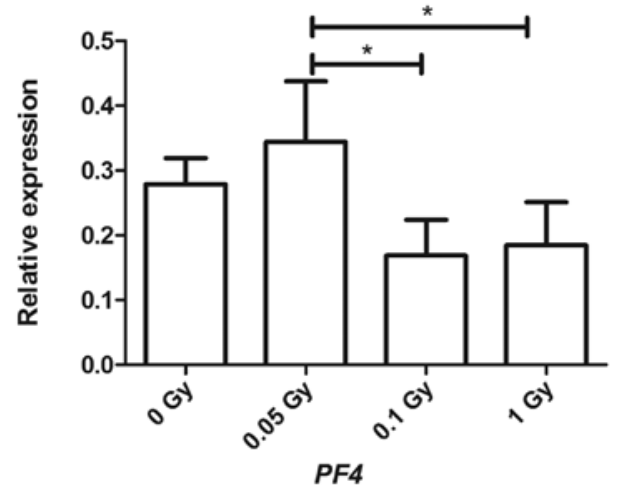

Figure 4. PF4 gene was downregulated in response to 0.1 and 1 Gy X-rays. Comparative qRT-PCR results of the PF4 gene in response to low and high doses of ionizing radiation. The chart shows the mean values and the standard error of the mean. Statistical significance was calculated by applying the Friedman test followed by Dunn's multiple comparison test. $\mathrm{P}<0.05$ was considered statistically significant. ${ }^{*} \mathrm{P}<0.05$. the activation of MAPKs. These are serine/threonine protein kinases that are activated subsequent to phosphorylation. The three major MAPKs are p38, ERK and JNK. Our results showed an upregulation of the phosphorylated form, thus the activated form, of the three MAPKs in response to 0.05 Gy (Fig. 3B, C and D). By contrast, higher doses (0.1 and $1 \mathrm{~Gy}$ ) exhibited a downregulation of the three MAPKs (Fig. 3B, C and D). To investigate the responses of the MAPKs at different doses, we measured the levels of PF4 gene. PF4 (platelet factor 4) is known to induce the activation of MAPKs (p38, ERK and JNK) in monocytes. The expression levels were in agreement with the phosphorylation levels of the proteins; PF4 showed lower induction at 0.1 and $1 \mathrm{~Gy}$ with significant statistical difference between 0.05 Gy in comparison to 0.1 and $1 \mathrm{~Gy}$ (Fig. 4).

High doses of ionizing radiation induced damage responses and p53 activation. Samples irradiated with 1 Gy showed a 
A

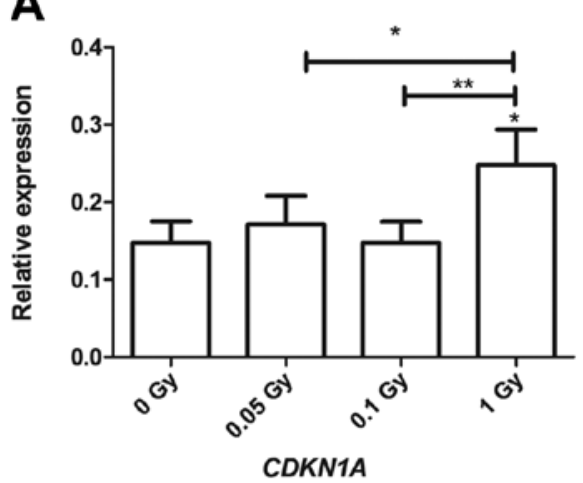

C

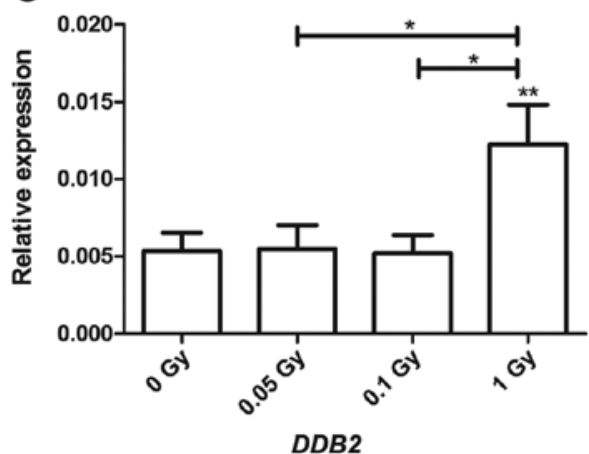

B

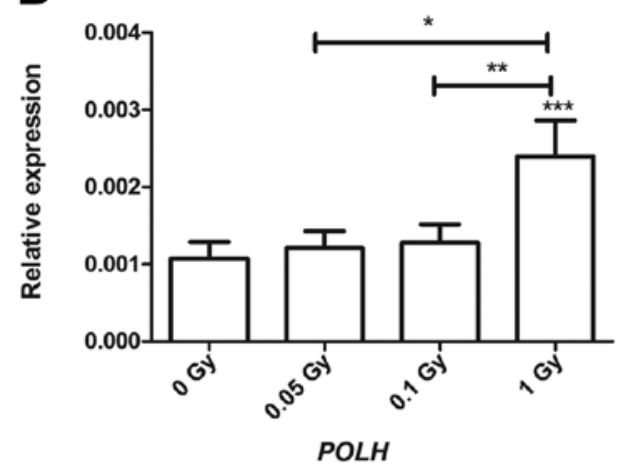

D

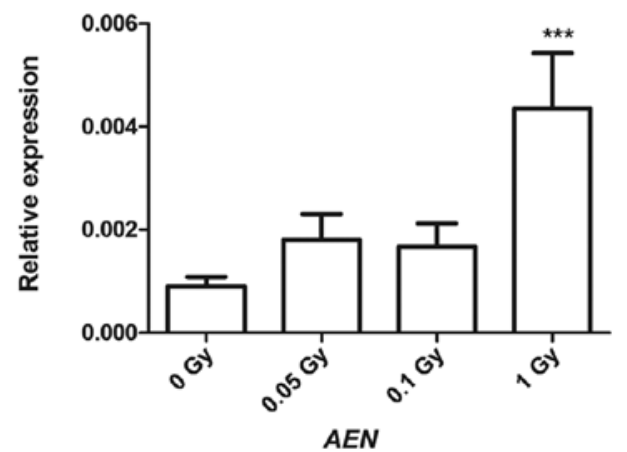

Figure 5. Cell cycle arrest, DNA damage and apoptosis were induced in response to 1 Gy X-rays. Comparative qRT-PCR results of (A) $C D K N 1 A$, (B) $P O L H$, (C) $D D B 2$ and (D) $A E N$ in response to low and high doses of ionizing radiation. The chart shows the mean values and the standard error of the mean. Statistical significance was calculated by applying the Friedman test followed by Dunn's multiple comparison test. $\mathrm{P}<0.05$ was considered statistically significant. ${ }^{*} \mathrm{P}<0.05 ;{ }^{* *} \mathrm{P}<0.005 ;{ }^{* * *} \mathrm{P}<0.0001$.

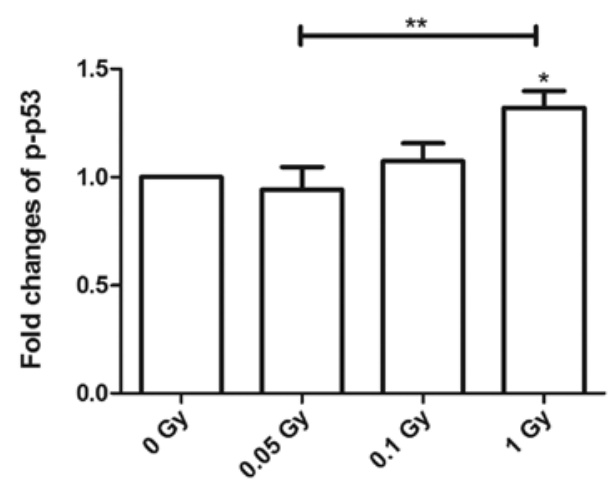

Figure 6. The phosphorylated form of p53 was induced in response to $1 \mathrm{~Gy}$ $\mathrm{X}$-rays. Fold changes of the phosphorylated form of the protein $\mathrm{p} 53$. The chart shows the mean values and the standard error of the mean. Statistical significance was calculated by applying one-way repeated measures ANOVA followed by Tukey's test for multiple comparisons. $\mathrm{P}<0.05$ was considered statistically significant. ${ }^{*} \mathrm{P}<0.05 ;{ }^{* *} \mathrm{P}<0.005$.

significant upregulation of the four genes involved in response to stress and damage (Fig. 5). These included cyclin-dependent kinase inhibitor 1A (CDKN1A), which plays a role in cycle arrest in response to DNA damage. It showed a statistical significance at $1 \mathrm{~Gy}$ compared to the sham-irradiated samples and between the different irradiation doses, i.e., between 0.05 and $1 \mathrm{~Gy}$, but also between 0.1 and 1 Gy (Fig. 5A). Additionally, polymerase DNA-direct $\eta(P O L H)$, a gene that plays a role in DNA repair in response to DNA damage was upregulated exclusively at 1 Gy. A statistically significant difference between 1 Gy in comparison to 0.05 and $0.1 \mathrm{~Gy}$ (Fig. 5B) was observed. Another DNA damage and repair gene, DNA damage binding protein-2 (DDB2), showed a similar expression profile to that of $P O L H$. It showed statistical significance in response to $1 \mathrm{~Gy}$ compared to the sham-irradiated samples with a statistical difference between $1 \mathrm{~Gy}$, compared to 0.05 and $0.1 \mathrm{~Gy}$ (Fig. 5C). The proapoptotic gene apoptosis enhancing nuclease $(A E N)$ showed an upregulatory trend at low doses $(0.05$ and $0.1 \mathrm{~Gy})$ without achieving statistical significance; however, it was significantly upregulated in response to 1 Gy (Fig. 5D). Furthermore, the phosphorylated form of p53 showed a significant upregulation in response to $1 \mathrm{~Gy}$ in comparison to the sham-irradiated samples, with a statistical significance between the induced levels at 0.05 and 1 Gy (Fig. 6).

\section{Discussion}

Gaining a better understanding of the mechanisms involved in cell and tissue responses to low doses of ionizing radiation is of increased interest as medical diagnostic and therapeutic applications involving $\mathrm{X}$-rays are significant contributory factors to the cumulative doses of the general population (18). Previously, whole genome expression studies showed that high doses of ionizing radiation predominantly induced DNA damage and apoptosis with p53 signaling playing a key role, while low doses induced mainly cytokine and chemokine signaling (5). To clarify the mechanisms behind this immune response induction, we investigated the activation of central immune pathways in the primary human monocytes following exposure 


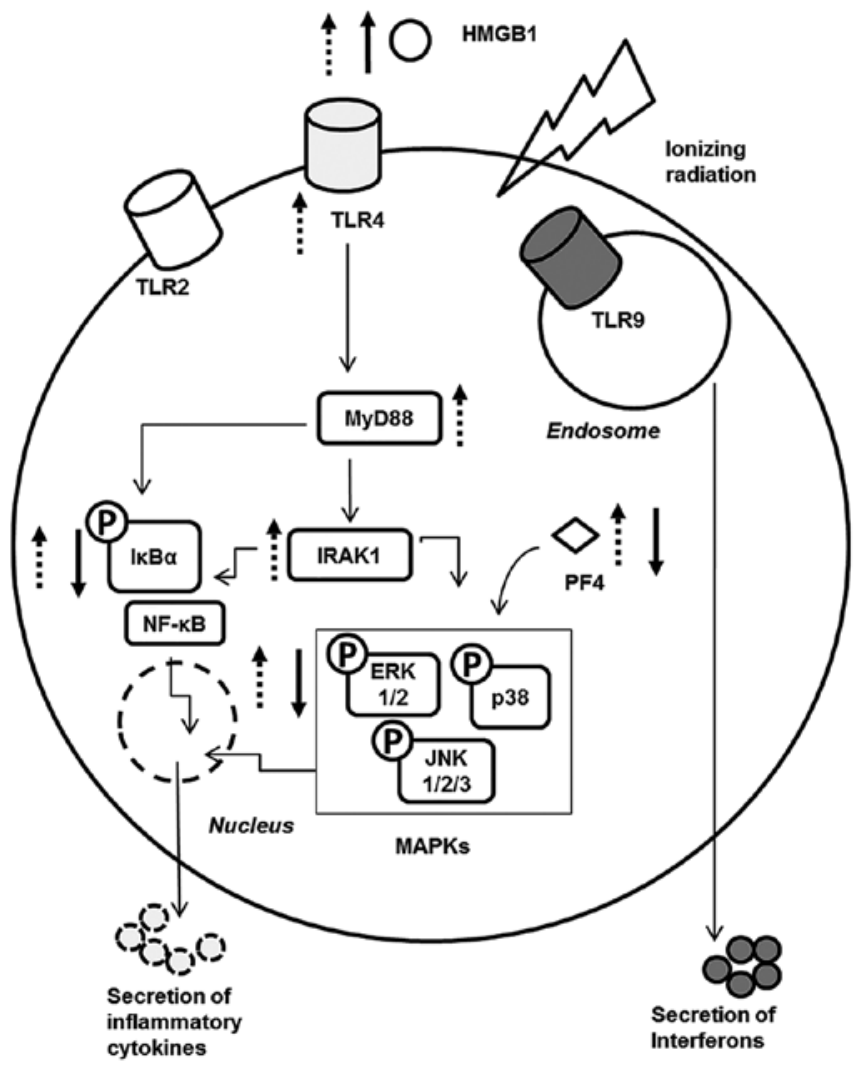

Figure 7. Schematic representation of the mechanisms involved in the induction of immune responses after exposure to a low (0.05 Gy) and a high dose ( $1 \mathrm{~Gy}$ ) of ionizing radiation. The dashed arrows represent $0.05 \mathrm{~Gy}$, while the complete arrows represent $1 \mathrm{~Gy}$. The direction of the arrows indicates the regulation status. Low doses of ionizing radiation induce TLR4 but not TLR2. TLR2, 4 and 9 are known to interact with HMGB1, leading to the activation of TLR signal transduction. The adapter molecule MyD88 plays a central role in the activation process, as it interacts with different molecules, such as IRAK1, which is involved in releasing NF- $\kappa \mathrm{B}$ transcription factor, thus facilitating its translocation to the nucleus via the phosphorylation of $\mathrm{I} \kappa \mathrm{B} \alpha$. Downstream IRAK1, MAPKs (p38, ERK and JNK) are activated, thus promoting pro-inflammatory signaling. PF4 is involved in the activation of MAPKs in monocytes. NF- $\kappa$ B and MAPKs signaling were activated at low but not high doses; activation of these pathways stimulate inflammatory and survival responses. HMGB1, high mobility group binding protein 1 TLR, toll-like receptor; MAPKs, mitogen-activated protein kinases; PF4, platelet factor 4 .

to low and high doses of ionizing radiation. Our results showed immune-stimulatory and pro-survival responses at low doses via the involvement of the TLRs, MAP kinases and positive regulation $\mathrm{NF}-\kappa \mathrm{B}$ signaling pathways. On the other hand, a dose of $1 \mathrm{~Gy}$ showed less involvement of the pro-survival and pro-inflammatory pathways, of NF- $\kappa \mathrm{B}$ signaling and MAP kinase activation; however, damage responses were induced.

TLR4 plays a crucial role in activating monocytes and in initiating an innate immune response. TLR4 detects lipopolysaccharides (LPS) and mediates its signal transduction via CD14 receptors (10). Our results have shown that low doses of ionizing radiation induced the activation of TLR4, while there was no significant change in its expression following exposure to 1 Gy (Fig. 1A). Previously, it has been reported that doses in the range of 0.075-2 Gy increased the expression of TLR4-MD2 in mouse macrophages (12). Additionally, TLR4 was shown to be involved in the induction of radia- tion resistance (19), cell proliferation and the promotion of radiation-induced lymphomas (20), as well as the promotion of reactive oxygen and nitrogen species (ROS/RNS) causing the aggravation of chronic inflammatory diseases (21). One of the novel activators of TLR4 is HMGB1 (22). The expression of HMGB1 increased significantly at all doses (Fig. 1B). Principally, HMGB1 is a nuclear DNA binding protein that plays a role in the stabilization of chromosomes and transcription regulation. However, recently, the role of HMGB1 extended to include inflammation and necrosis (22). Activated monocytes and macrophages secrete HMGB1 and promote immune-stimulatory signaling (23). On the other hand, damaged cells 'passively' release HMGB1 into the extracellular environment (24), which could explain the dose-dependent response demonstrated in our data (Figs. 1B and 7). Another TLR that is known to interact with HMGB1 is TLR2 (25). Results of the present study did not show any significant changes in the expression of TLR2 at all doses (Figs. 1C and 7). It has been reported that the interaction between HMGB1 and TLR2 is structurally and mechanistically different to TLR4 as TLR 2 interacts only with nucleosome-bound HMGB1 proteins (26).

Bacterial CpG DNA sequences and DAMPs, including HMGB1, activate TLR9 $(10,13)$. Our results showed an upregulatory trend of TLR9 at all doses (Figs. 1D and 7). Ermakov et al $(27,28)$ showed that irradiation of lymphocytes and endothelial cells with 0.1 Gy led to the secretion of extracellular DNA fragments, which promotes the activation of TLR9. Furthermore, they reported that these DNA fragments, along with TLR9, played a role in bystander signaling between irradiated and non-irradiated cells.

Downstream of the TLR signaling, the MyD88 adapter molecule is known to be crucial for the secretion of proinflammatory cytokines, such as IL-12 and IL-18 (29,30). MyD88 is involved in the downstream signaling of TLR4 and TLR9. It showed statistically significant overexpression in response to $0.05 \mathrm{~Gy}$, while the two other doses (0.1 and $1 \mathrm{~Gy}$ ) were upregulatied without reaching statistical significance (Figs. 2A and 7).

Following activation, MyD88 interacts with the IRAK1 molecule, which plays a role in releasing the NF- $\kappa \mathrm{B}$ transcription factor via the phosphorylation of the sequestering protein $\mathrm{I} \kappa \mathrm{B} \alpha$ (31). IRAK 1 was overexpressed significantly at 0.05 and $0.1 \mathrm{~Gy}$ but not at $1 \mathrm{~Gy}$ (Fig. 2B). Furthermore, the downstream molecule I $\mathrm{B} \alpha$ was upregulated by 2 - and 2.5 -fold changes at 0.05 and $0.1 \mathrm{~Gy}$, respectively (Figs. 3A). By contrast, the phosphorylation levels of I $\mathrm{B} \alpha$ exhibited a dephosphorylation pattern in response to $1 \mathrm{~Gy}$ (Fig. 3A). The positive regulation of $\mathrm{NF}-\kappa \mathrm{B}$ signaling at low doses indicates the promotion of pro-survival and pro-inflammatory responses, whereas the suppression of $\mathrm{NF}-\kappa \mathrm{B}$ signaling at high doses indicates a proapoptotic response (16).

MAPKs such as p38, ERK $1 / 2$ and JNK, are activated downstream of the HMGB1-TLR4-MyD88-IRAK1 signaling axis (15). Our results showed increased fold changes of phosphorylation, thus activation, of the three MAPKs at 0.05 Gy (Fig. 3B-D). However, and in contrast to our expectations, the phosphorylation levels did not show any increased fold changes at $0.1 \mathrm{~Gy}$, which showed a similar response to that of 1 Gy (Fig. 3B-D). Previously, we have demonstrated that 


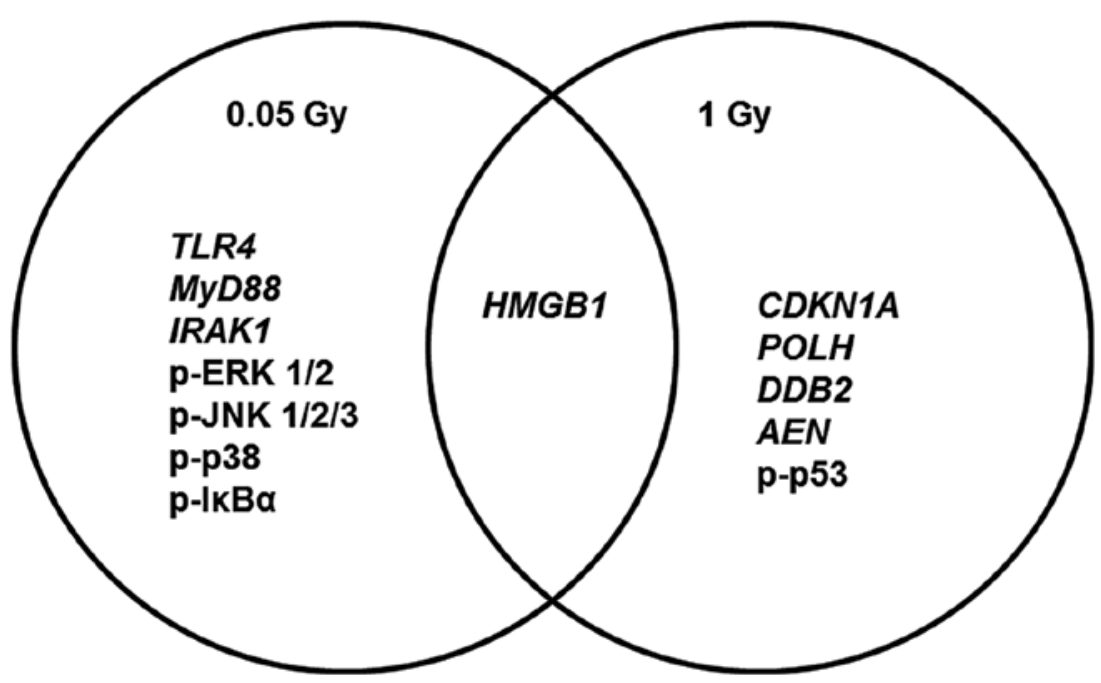

Figure 8. Venn diagram comparing different genes and proteins induced in response to low ( $0.05 \mathrm{~Gy})$ and high (1 Gy) doses of ionizing radiation.

PF4 gene was upregulated in whole blood samples exposed to low doses of ionizing radiation (5). PF4 encodes for the platelet factor-4 protein, a chemokine expressed in platelets, $\mathrm{T}$ cells, monocytes, endothelial and muscle cells (32). In monocytes, it induces phagocytosis and activates MAPK family proteins (p38, ERK and JNK) leading to ROS formation (33). Our results showed a significant downregulation in PF4 at 0.1 and 1 Gy compared to 0.05 Gy (Figs. 4 and 7). This result is in agreement with the activated MAPK levels measured at the protein levels. This suggests a role of PF4 in the activation or suppression of MAPKs in response to ionizing radiation.

Previously, we have reported that MAPK cascades play a central role in whole blood samples irradiated with 0.05 Gy (5). In addition to that, studies on exposure to low doses have reported a proliferative response through the activation of ERK $1 / 2$, p38 and JNK $(34,35)$. A study by Kim et al (36) showed that in normal diploid cells, there is a transient phosphorylation of ERK $1 / 2$ and p38 but not JNK 1/2 in response to 0.05 Gy of $\gamma$-irradiation, which was accompanied with the activation of several downstream transcription factors that promote growth signaling, including Elk-1, p90RSK and ATF-2. Another study performed by Liang et al (37) showed that MAPK proteins c-Raf, ERK 1/2 and MEK 1/2 were activated in mesenchymal stem cells in response to 0.075 Gy of X-rays, thus a proliferative response was concluded.

In contrast to results obtained at the exposure dose of $0.05 \mathrm{~Gy}$, our results showed suppressed MAPKs levels after exposure to $1 \mathrm{~Gy}$ (Fig. 3B-D). Tsukimoto et al (38) demonstrated that increased levels of MAPK phosphatase-1 (MKP-1) promote dephosphorylation of p38 and ERK1/2 in the macrophage cell line (RAW2674.7) irradiated with $0.5-1$ Gy $\gamma$-irradiation. In addition to that, studies performed by Lödermann et al (39) demonstrated a decreased induction of $\mathrm{NF}-\kappa \mathrm{B}$ and p38 in response to $0.5-0.7 \mathrm{~Gy}$ of $\gamma$-irradiation.

However, our results demonstrated that the damage responses were very prominent in response to $1 \mathrm{~Gy}$. CDKN1A (Fig. 5A) a cell cycle arrest inducer gene; $P O L H$ (Fig. 5B) and $D D B 2$ (Fig. 5C), DNA damage and repair genes; and $A E N$, (Fig. 5D) a pro-apoptotic gene, were shown to be exclusively induced in response to $1 \mathrm{~Gy}$. These genes were extensively validated in response to ionizing radiation (40-44) and shown to be activated in response to p53 signaling (45), where as our results showed upregulation in the phosphorylated form of p53 in response to 1 Gy (Fig. 6). Altogether, the 1 Gy results demonstrate immuno-suppressive and damaging responses.

In conclusion, we have demonstrated that monocytes exposed to low doses of ionizing induce immune-stimulatory and pro-survival responses, while those exposed to high doses induced immuno-suppressive and damaging responses. Low-dose responses were transduced via the TLR4 signaling axis, i.e., HMGB1-TLR4-MyD88-IRAK1-MAPKs-NF- $\mathrm{B}$, which showed a particular upregulation in response to $0.05 \mathrm{~Gy}$. By contrast, the same axis did not demonstrate similar induction in response to $1 \mathrm{~Gy}$. Of note, 0.1 Gy showed a 'borderline' immune-stimulatory response between low and high doses, i.e., TLRs and NF- $\mathrm{B}$ signaling were upregulated but not MAPKs. On other hand, damaged genes and p53 were exclusively involved in response to $1 \mathrm{~Gy}$ but not at lower doses (Fig. 8). In view of these results, we clearly address the necessity to consider the health risks that can be induced in response to low doses of ionizing radiation, with the TLR, NF- $\kappa \mathrm{B}$ and MAPK signaling pathways being involved in tumor proliferation, autoimmunity and chronic inflammation $(15,16,22,46)$. Furthermore, there is accumulation of evidence that has demonstrated an increased cancer risk due to exposure to low doses from medical diagnostics. Thus, there is a need for the re-evaluation of the radiation protection measures within the medical field.

\section{Acknowledgements}

The authors are thankful to all the blood donors and SCK-CEN medical staff who kindly accepted to participate in our study. In addition, we appreciate Dr P. Willems [Federal Agency for Nuclear Control (FANC), Belgium] for the useful scientific discussions carried out through the preparation of the study. H. El-Saghire is supported by a doctoral SCK-CEN/Ghent University grant. This study was funded by the FANC CT-SCAN contract (CO-90-09-2329-00) and by the FP7 EU EPI-CT contract (grant agreement 269912). 


\section{References}

1. Hall EJ and Brenner DJ: Cancer risks from diagnostic radiology. Br J Radiol 81: 362-378, 2008.

2. Pearce MS, Salotti JA, Little MP, et al: Radiation exposure from CT scans in childhood and subsequent risk of leukaemia and brain tumours: a retrospective cohort study. Lancet 380: 499-505, 2012.

3. Pernot $\mathrm{E}$, Hall J, Baatout $\mathrm{S}$, et al: Ionizing radiation biomarkers for potential use in epidemiological studies. Mutat Res 751: 258-286, 2012.

4. Shah DJ, Sachs RK and Wilson DJ: Radiation-induced cancer: a modern view. Br J Radiol 85: e1166-e1173, 2012.

5. El-Saghire H, Thierens H, Monsieurs P, Michaux A Vandevoorde $\mathrm{C}$ and Baatout S: Gene set enrichment analysis highlights different gene expression profiles in whole blood samples X-irradiated with low and high doses. Int J Radiat Biol 89: 628-638, 2013.

6. Hume DA: The mononuclear phagocyte system. Curr Opin Immunol 18: 49-53, 2006.

7. Bauer M, Goldstein M, Christmann M, Becker H, Heylmann D and Kaina B: Human monocytes are severely impaired in base and DNA double-strand break repair that renders them vulnerable to oxidative stress. Proc Natl Acad Sci USA 108: 21105-21110, 2011.

8. Kawai T and Akira S: The role of pattern-recognition receptors in innate immunity: update on Toll-like receptors. Nat Immunol 11: 373-384, 2010.

9. Krutzik SR, Tan B, Li H, et al: TLR activation triggers the rapid differentiation of monocytes into macrophages and dendritic cells. Nat Med 11: 653-660, 2005.

10. Piccinini AM and Midwood KS: DAMPening inflammation by modulating TLR signalling. Mediators Inflamm 2010: 672395 , 2010.

11. Kawai T and Akira S: Toll-like receptors and their crosstalk with other innate receptors in infection and immunity. Immunity 34 : 637-650, 2011

12. Shan YX, Jin SZ, Liu XD, Liu Y and Liu SZ: Ionizing radiation stimulates secretion of pro-inflammatory cytokines: doseresponse relationship, mechanisms and implications. Radiat Environ Biophys 46: 21-29, 2007.

13. Ivanov S, Dragoi AM, Wang X, et al: A novel role for HMGB1 in TLR9-mediated inflammatory responses to CpG-DNA. Blood 110: 1970-1981, 2007.

14. Menendez D, Shatz M, Azzam K, Garantziotis S, Fessler MB and Resnick MA: The Toll-like receptor gene family is integrated into human DNA damage and p53 networks. PLoS Genet 7: e1001360, 2011.

15. Cargnello $M$ and Roux PP: Activation and function of the MAPKs and their substrates, the MAPK-activated protein kinases. Microbiol Mol Biol Rev 75: 50-83, 2011.

16. Hayden MS and Ghosh S: NF- $\kappa B$, the first quarter-century: remarkable progress and outstanding questions. Genes Dev 26 : 203-234, 2012

17. Pfaffl MW: A new mathematical model for relative quantification in real-time RT-PCR. Nucleic Acids Res 29: e45, 2001.

18. UNSCEAR: Report of the fifty seventh session: Summary of low-dose radiation effects on health. 2010.

19. Liu C, Zhang C, Mitchel RE, et al: A critical role of toll-like receptor 4 (TLR4) and its' in vivo ligands in basal radio-resistance. Cell Death Dis 4: e649, 2013.

20. Liu C, Gao F, Li B, et al: TLR4 knockout protects mice from radiation-induced thymic lymphoma by downregulation of IL6 and miR-21. Leukemia 25: 1516-1519, 2011.

21. Lucas K and Maes M: Role of the Toll like receptor (TLR) radical cycle in chronic inflammation: possible treatments targeting the TLR4 pathway. Mol Neurobiol 48: 190-204, 2013.

22. Schaue D and McBride WH: Links between innate immunity and normal tissue radiobiology. Radiat Res 173: 406-417, 2010.

23. Wang FP, Li L, Li J, Wang JY, Wang LY and Jiang W: High mobility group box-1 promotes the proliferation and migration of hepatic stellate cells via TLR4-dependent signal pathways of PI3K/Akt and JNK. PLoS One 8: e64373, 2013.

24. Andersson U and Tracey KJ: HMGB1 is a therapeutic target for sterile inflammation and infection. Annu Rev Immunol 29: 139-162, 2011

25. Weng H, Deng Y, Xie Y, Liu H and Gong F: Expression and significance of HMGB1, TLR4 and NF- $\mathrm{B}$ p 65 in human epidermal tumors. BMC Cancer 13: 311, 2013.
26. Mittal D, Saccheri F, Venereau E, Pusterla T, Bianchi ME and Rescigno M: TLR4-mediated skin carcinogenesis is dependent on immune and radioresistant cells. EMBO J 29: 2242-2252, 2010.

27. Ermakov AV, Konkova MS, Kostyuk SV, Egolina NA, Efremova LV and Veiko NN: Oxidative stress as a significant factor for development of an adaptive response in irradiated and nonirradiated human lymphocytes after inducing the bystander effect by low-dose X-radiation. Mutat Res 669: 155-161, 2009.

28. Kostyuk SV, Ermakov AV, Alekseeva AY, et al: Role of extracellular DNA oxidative modification in radiation induced bystander effects in human endotheliocytes. Mutat Res 729: 52-60, 2012.

29. Ichikawa S, Miyake M, Fujii R and Konishi Y: MyD88 associated ROS generation is crucial for Lactobacillus induced IL-12 production in macrophage. PLoS One 7: e35880, 2012.

30. Fiedler K, Kokai E, Bresch S and Brunner C: MyD88 is involved in myeloid as well as lymphoid hematopoiesis independent of the presence of a pathogen. Am J Blood Res 3: 124-140, 2013.

31. Windheim M, Stafford M, Peggie $M$ and Cohen P: Interleukin-1 (IL-1) induces the Lys63-linked polyubiquitination of IL-1 receptor-associated kinase 1 to facilitate NEMO binding and the activation of IkappaBalpha kinase. Mol Cell Biol 28: 1783-1791, 2008

32. Xiao Z, Visentin GP, Dayananda KM and Neelamegham S: Immune complexes formed following the binding of anti-platelet factor 4 (CXCL4) antibodies to CXCL4 stimulate human neutrophil activation and cell adhesion. Blood 112: 1091-1100, 2008.

33. Kasper B, Brandt E, Brandau S and Petersen F: Platelet factor 4 (CXC chemokine ligand 4) differentially regulates respiratory burst, survival, and cytokine expression of human monocytes by using distinct signaling pathways. J Immunol 179: 2584-2591, 2007.

34. Narang $\mathrm{H}$ and Krishna M: Mitogen-activated protein kinases: specificity of response to dose of ionizing radiation in liver. J Radiat Res 45: 213-220, 2004.

35. Rizvi A, Pecaut MJ, Slater JM, Subramaniam S and Gridley DS: Low-dose $\gamma$-rays modify CD4(+) T cell signalling response to simulated solar particle event protons in a mouse model. Int J Radiat Biol 87: 24-35, 2011.

36. Kim CS, Kim JM, Nam SY, et al: Low-dose of ionizing radiation enhances cell proliferation via transient ERK1/2 and p38 activation in normal human lung fibroblasts. J Radiat Res 48: 407-415, 2007.

37. Liang X, So YH, Cui J, et al: The low-dose ionizing radiation stimulates cell proliferation via activation of the MAPK/ERK pathway in rat cultured mesenchymal stem cells. J Radiat Res 52: 380-386, 2011.

38. Tsukimoto M, Homma T, Mutou Y and Kojima S: 0.5 Gy gamma radiation suppresses production of TNF-alpha through up-regulation of MKP-1 in mouse macrophage RAW264.7 cells. Radiat Res 171: 219-224, 2009.

39. Lödermann B, Wunderlich R, Frey S, et al: Low dose ionising radiation leads to a NF- $\kappa \mathrm{B}$ dependent decreased secretion of active IL-1 $\beta$ by activated macrophages with a discontinuous dose-dependency. Int J Radiat Biol 88: 727-734, 2012.

40. Turtoi A, Brown I, Oskamp D and Schneeweiss FH: Early gene expression in human lymphocytes after gamma-irradiation-a genetic pattern with potential for biodosimetry. Int J Radiat Biol 84: 375-387, 2008.

41. Brengues M, Paap B, Bittner M, et al: Biodosimetry on small blood volume using gene expression assay. Health Physics 98: 179-185, 2010.

42. Kabacik S, Mackay A, Tamber N, et al: Gene expression following ionising radiation: identification of biomarkers for dose estimation and prediction of individual response. Int $\mathrm{J}$ Radiat Biol 87: 115-129, 2011.

43. Mayer C, Popanda O, Greve B, et al: A radiation-induced gene expression signature as a tool to predict acute radiotherapyinduced adverse side effects. Cancer Lett 302: 20-28, 2011.

44. Riecke A, Rufa CG, Cordes M, Hartmann J, Meineke V and Abend M: Gene expression comparisons performed for biodosimetry purposes on in vitro peripheral blood cellular subsets and irradiated individuals. Radiat Res 178: 234-243, 2012.

45. Rashi-Elkeles S, Elkon R, Shavit S, et al: Transcriptional modulation induced by ionizing radiation: $\mathrm{p} 53$ remains a central player. Mol Oncol 5: 336-348, 2011.

46. Wagner EF and Nebreda AR: Signal integration by JNK and p38 MAPK pathways in cancer development. Nat Rev Cancer 9: 537-549, 2009. 